\title{
Modes and nodes explain the mechanism of action of vortioxetine, a multimodal agent (MMA): actions at serotonin receptors may enhance downstream release of four pro-cognitive neurotransmitters
}

\author{
Stephen M. Stahl
}

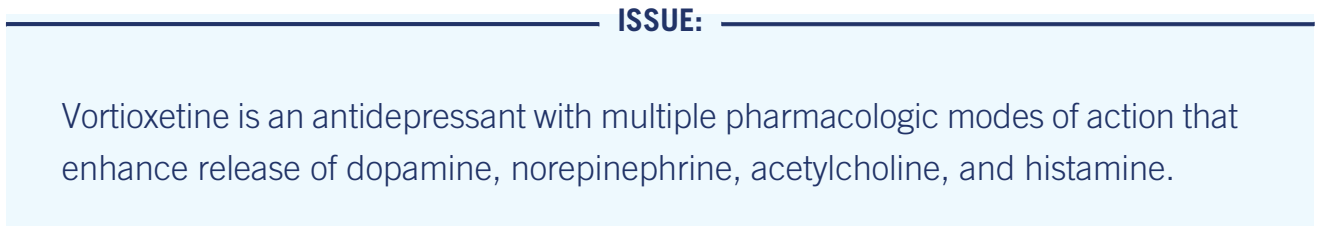

\section{Take-Home Points}

- Vortioxetine has a complex mechanism of action that includes not only inhibition of serotonin (5HT) transporters (SERT), but also direct actions at multiple $5 \mathrm{HT}$ receptor subtypes (5HT1A, 5HT1B, 5HT1D, 5HT3, and 5HT7 receptors).

- Vortioxetine has direct actions at 5HT1A and 5HT1B heteroreceptors that may explain in part how vortioxetine causes the downstream release of dopamine (DA), norepinephrine (NE), histamine (HA), and acetylcholine (ACh).

- Enhanced release of neurotransmitters in the prefrontal cortex and hippocampus could hypothetically help to explain vortioxetine's antidepressant actions and unique procognitive properties in patients with major depression.

Vortioxetine is a "multimodal" agent that simultaneously acts at 6 pharmacologic targets with 3 modes of action (Figure 1) ${ }^{1-5}$ :

1. Inhibition of the serotonin (5HT) transporter or SERT

2. Actions at several G-protein linked receptors (agonist actions at 5HT1A receptors, partial agonist actions at 5HT1B receptors, antagonist actions at 5HT1D and 5HT7 receptors)

3. Inhibition of a ligand-gated ion channel (the 5HT3 receptor)
We have previously described the mechanisms whereby vortioxetine's actions at 5HT receptors work together to enhance the release of $5 \mathrm{HT}^{6}{ }^{6}$ glutamate, ${ }^{7}$ acetylcholine $(\mathrm{ACh}){ }^{8}$ and norepinephrine $(\mathrm{NE})^{8}$ and to inhibit the release of GABA (gamma amino butyric acid). ${ }^{7}$ Here we discuss how the interaction of vortioxetine at populations of 5HT1A and 5HT1B receptors (Figure 1) may also hypothetically contribute to the release of $\mathrm{ACh}$ and $\mathrm{NE}$ and furthermore lead to release of dopamine (DA) and histamine (HA) in the prefrontal cortex (Figures 2 and 3). Enhanced release of neurotransmitters may theoretically "tune" malfunctioning brain circuits. ${ }^{6-11}$ Specifically, enhanced release of 5HT, $\mathrm{NE}, \mathrm{DA}, \mathrm{ACh}$, and HA by vortioxetine could theoretically improve the efficiency of information processing in maladaptive brain circuits by facilitating long-term potentiation, synaptic plasticity, and enhanced pyramidal neuron activity leading to improvement not only of mood but also of cognitive symptoms in major depressive disorder.

Improving the Efficiency of Information Processing in Neuronal Networks by Enhancing the Release of Key Neurotransmitters

In this series of articles on the mechanism of action of vortioxetine, we have discussed how the many modes of action of this agent interact with multiple 5HT receptor subtypes localized at various critical nodes that connect $5 \mathrm{HT}$ neurons to a network of several other neurons. ${ }^{6-8}$ 


\section{BRAINSTORMS-Clinical Neuroscience Update}

Figure 1. Icon of vortioxetine showing its 6 pharmacologic mechanisms. Highlighted here are 5HT1A agonism and 5HT1B partial agonism, potentially linked to vortioxetine's actions of enhancing the release of NE, DA, ACh, and HA.

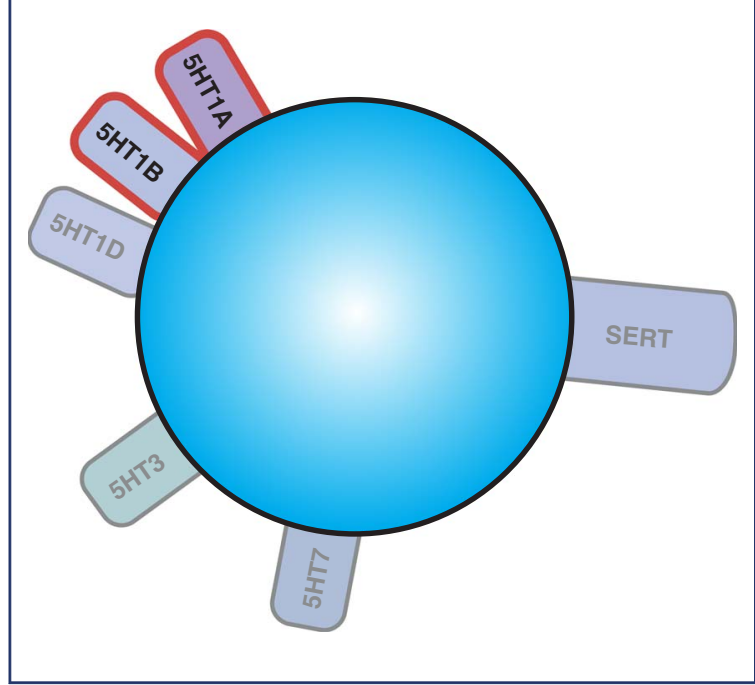

Actions of neurotransmitters, drugs, and psychiatric illnesses can be understood not only within "microcircuits" - eg, connections between a presynaptic 5HT neuron and a postsynaptic site-but also within "macrocircuits," eg, where serotonergic neurons are part of a neuronal network that connects many neurons with each other. $^{6-13}$ The sites where neurons connect with each other are also called the "nodes" of a neuronal network, and in the case of 5HT nodes, are linked by many different 5HT receptor subtypes. Not only does 5HT acting at these nodes regulate its own release, but it modulates the release of every major neurotransmitter. $^{1-8,12,13}$ We have discussed how this happens with $5 \mathrm{HT}$ itself at 5HT neurons regulated by numerous autoreceptors, ${ }^{6}$ with glutamate and GABA at connecting neurons receiving $5 \mathrm{HT}$ input especially at many different $5 \mathrm{HT}$ receptors, ${ }^{7}$ and with $\mathrm{ACh}, \mathrm{NE}$, and $5 \mathrm{HT}$ via actions at $5 \mathrm{HT} 3$ receptors. $^{8}$ Here we describe 2 additional ways in which $5 \mathrm{HT}$ could theoretically modulate neurotransmitter release at 5HT1A and 5HT1B heteroreceptors in the prefrontal cortex, and specifically how vortioxetine acting at these sites could potentially enhance the release of DA, NE, ACh, and HA (Figures 2 and 3).

\section{HT1A Postsynaptic Heteroreceptors in Prefrontal Cortex: Potential Modulation of DA, ACh, and NE Release}

We have previously discussed the role of 5HT1A presynaptic autoreceptors located in the midbrain
Figure 2A. Possible regulation of NE, DA, and ACh release by cortical postsynaptic serotonin $1 \mathrm{~A}$ heteroreceptors. GABA release is inhibited by 5HT1A input to GABAergic interneurons that in turn innervate the presynaptic nerve terminals of NE, DA, and ACh neurons.

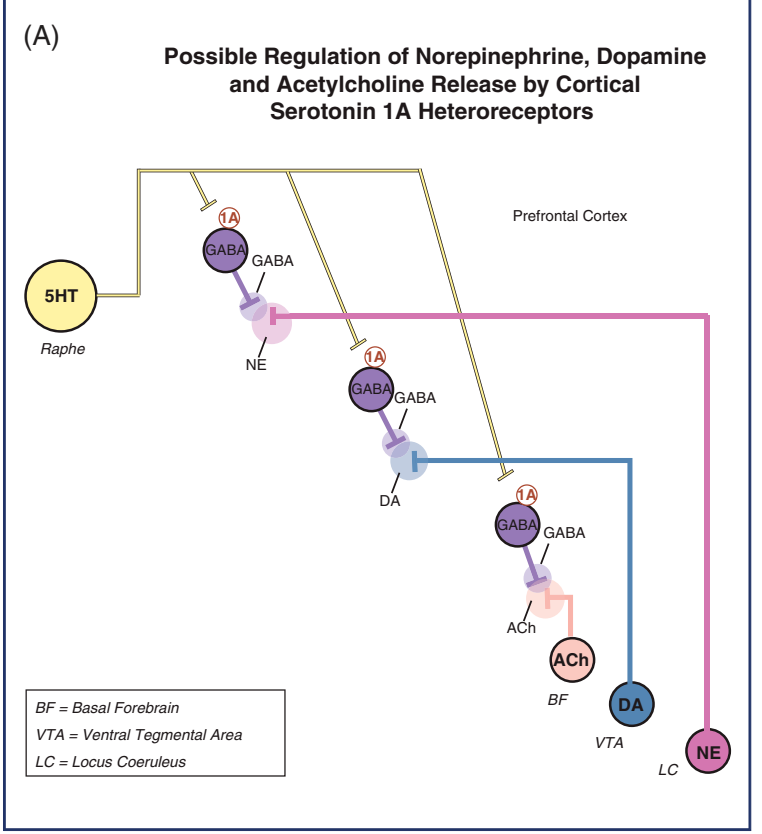

raphe and on soma and dendrites of 5HT neurons. ${ }^{6}$ These receptors desensitize over time when either SSRIs or vortioxetine are given, leading to disinhibition of 5 HT release ${ }^{14-17}$ By contrast, when these same 5HT1A receptors are localized on postsynaptic sites, they do not seem to desensitize over time after the administration of SSRIs or vortioxetine, but they regulate the release of many other neurotransmitters. ${ }^{11,14-17}$ 5HT1A postsynaptic receptors are inhibitory, so stimulating them either indirectly after SERT blockade by an SSRI or directly with a 5HT1A agonist such as vortioxetine will inhibit the firing of that neuron. ${ }^{11,14-17}$ We have already discussed how this specifically inhibits certain GABA interneurons regulating glutamate release. ${ }^{7,8}$ Here we discuss and illustrate the possible actions of $5 \mathrm{HT}$ at a different population of GABA neurons in prefrontal cortex, namely, those that possibly regulate the release of $\mathrm{NE}, \mathrm{DA}$, and $\mathrm{ACh}$ at their presynaptic nerve terminals (Figure 2A). GABA release is inhibited by 5HT1A input to these GABAergic interneurons, so when vortioxetine stimulates these 5HT1A receptors, this could potentially disinhibit the release of $\mathrm{ACh},{ }^{18,19} \mathrm{NE}^{20,21}$ and $\mathrm{DA}^{22,23}$ from their nerve terminals in the prefrontal cortex (Figure 2B). 


\section{BRAINSTORMS-Clinical Neuroscience Update}

Figure 2B. Possible disinhibition of NE, DA, and ACh release by vortioxetine acting at cortical postsynaptic serotonin 1A heteroreceptors. Vortioxetine directly stimulates 5HT1A receptors on GABA interneurons innervating the presynaptic nerve terminals of NE, DA, and ACh neurons. This could potentially disinhibit (enhance) the release of DA, NE, and ACh from their nerve terminals in the prefrontal cortex.

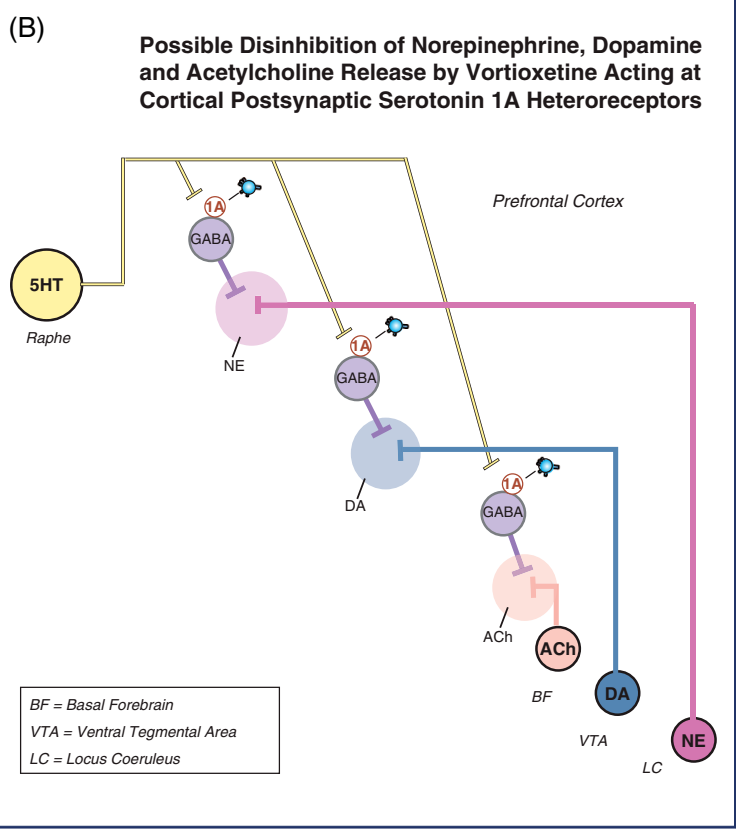

Not shown because of the lack of data-but still a theoretical possibility-is the same regulatory system for histamine. Figures 2A and 2B are just hypothetical wiring diagrams that are consistent with the available data, but require much further investigation to confirm that this is how 5HT1A agonists actually increase ACh, $\mathrm{NE}$, and DA release.

\section{HT1B Postsynaptic Heteroreceptors in the Prefrontal Cortex: Potential Modulation of DA, ACh, NE, and HA Release}

We have also previously discussed the role of 5HT1B autoreceptors that are located on $5 \mathrm{HT}$ nerve terminals and inhibit 5HT release. ${ }^{6}$ When $5 \mathrm{HT}$ occupies these receptors, it inhibits further $5 \mathrm{HT}$ release from those 5 HT nerve terminals. ${ }^{6,11,12}$ To the extent that vortioxetine blocks these $5 \mathrm{HT} 1 \mathrm{~B}$ receptors as a partial agonist or functional antagonist, the opposite effect occurs, namely enhanced 5HT release, as discussed previously. ${ }^{6}$ We have also previously discussed the possible role
Figure 3A. Possible regulation of several pro-cogntive neurotransmitters by cortical serotonin $1 \mathrm{~B}$ heteroreceptors. A subpopulation of $5 \mathrm{HT} 1 \mathrm{~B}$ receptors may be localized directly upon presynaptic nerve terminals of $\mathrm{NE}, \mathrm{DA}, \mathrm{ACh}$, and HA neurons. These are heteroreceptors because they are not located on $5 \mathrm{HT}$ neurons. These $5 \mathrm{HT} 1 \mathrm{~B}$ receptors are postsynaptic relative to their $5 \mathrm{HT}$ neurons, yet they are also presynaptic relative to $\mathrm{NE}, \mathrm{DA}, \mathrm{ACh}$, and $\mathrm{HA}$ neurons. $5 \mathrm{HT} 1 \mathrm{~B}$ receptors are inhibitory and $5 \mathrm{HT}$ reduces the release of neurotransmitter from these neurons.

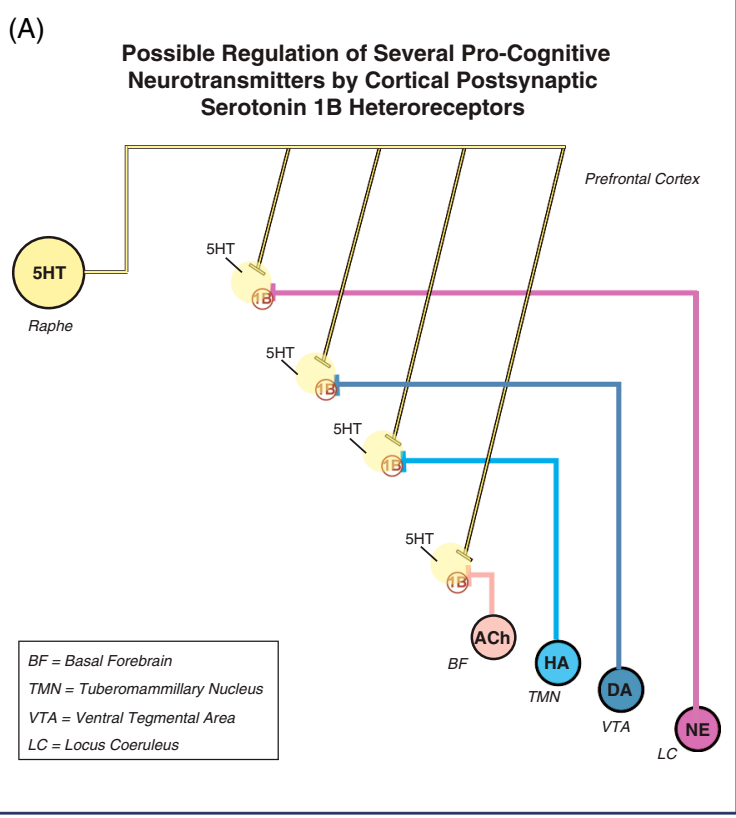

of 5HT1B heteroreceptors that may be localized on some GABAergic interneurons and may regulate GABA release. $^{7}$

Here, however, we discuss and illustrate the actions of $5 \mathrm{HT}$ at a different $5 \mathrm{HT} 1 \mathrm{~B}$ receptor that is possibly localized directly upon the nerve terminals of neurons that release NE, DA, ACh, and HA (Figure 3A). 12,24,25 Although the microanatomy is still being worked out, blockade of postsynaptic 5HT1B heteroreceptors on presynaptic nerve terminals could theoretically be another mechanism whereby $\mathrm{ACh}, \mathrm{NE}, \mathrm{DA}$, and HA release is enhanced by vortioxetine (Figure $3 \mathrm{~B}$ ). ${ }^{8,10}$ This is somewhat speculative and is not yet a proven mechanism for regulating the release of these neurotransmitters, but is consistent with the known effects of vortioxetine enhancing the release of ACh, NE, DA, and HA. Few if any other agent has the broad neurotransmitter-releasing properties of vortioxetine, and a full explanation of the mechanism of this release will require a more complete clarification of the various 


\section{BRAINSTORMS - Clinical Neuroscience Update}

Figure 3B. Possible disinhibition of several pro-cognitive neurotransmitters by vortioxetine acting at cortical serotonin 1B heteroreceptors. Vortioxetine is a partial agonist at 5HT1B heteroreceptors and possibly a functional antagonist. Thus, occupancy of cortical 5HT1B heteroreceptors localized on NE, ACh, DA, and HA neurons by vortioxetine would theoretically disinhibit these neurons and enhance the release of their neurotransmitters.

(B)

Possible Disinhibition of Several Pro-Cognitive Neurotransmitters by Vortioxetine Acting at Cortical Postsynaptic Serotonin 1B Heteroreceptors

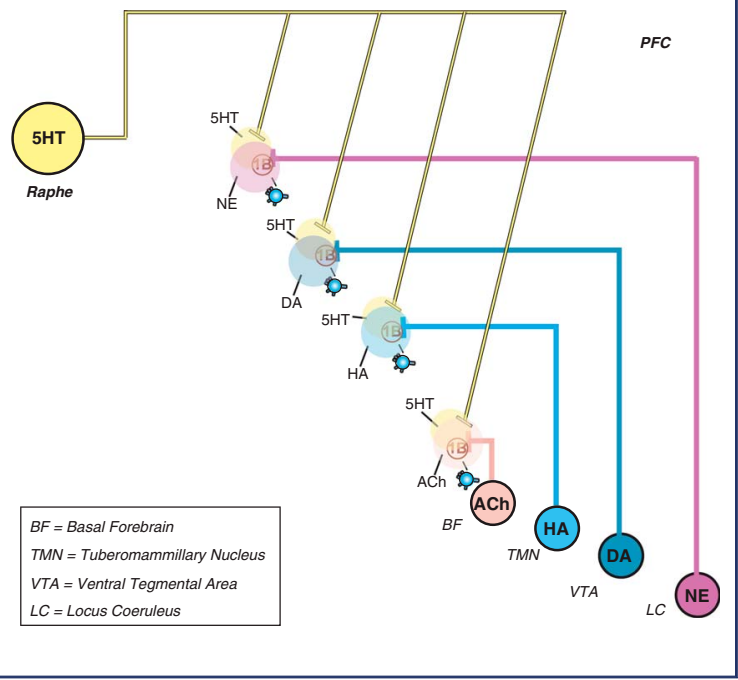

circuits involving 5HT receptors and other neurotransmitter systems. For example, some evidence suggests that stimulation of 5HT4 receptors may be in part responsible for 5HT's regulation of $\mathrm{HA}$ and $\mathrm{ACh}$ release. ${ }^{26}$ For now, mechanisms such as the one illustrated in Figure 3B remain only theories as to how vortioxetine could potentially disinhibit the release of $\mathrm{DA}, \mathrm{NE}, \mathrm{HA}$, and $\mathrm{ACh}$ in the prefrontal cortex and hippocampus.

\section{Clinical Implications}

Abnormal connectivity of brain circuits is theorized to cause symptoms of psychiatric disorders such as depression, and psychotropic drugs hypothetically reduce these symptoms by changing this connectivity, thus improving the efficiency of information processing in specific brain circuits. ${ }^{9,10}$ Since there are numerous symptoms in major depression, including both emotional symptoms and cognitive symptoms, it is likely that there are numerous networks with altered connectivity involved in major depression., ${ }^{9,10}$
Psychopharmacologic agents such as vortioxetine that can change the release of many neurotransmitters and in more than one site (ie, multiple modes of action at multiple nodes within brain networks) theoretically have the possibility to change multiple symptoms linked to multiple circuits. ${ }^{9,10}$ Thus, it is possible that such actions of vortioxetine can explain not only its antidepressant actions, ${ }^{4}$ but also its unique procognitive actions in patients with major depression. ${ }^{27-29}$

\section{References:}

1. Mørk A, Pehrson A, Brennum LT, et al. Pharmacological effects of Lu AA21004: a novel multimodal compound for the treatment of major depressive disorder. J Pharmacol Exp Ther. 2012; 340(3): 666-675.

2. Bang-Andersen $\mathrm{B}$, Ruhland $\mathrm{T}$, Jorgensen $\mathrm{M}$, et al. Discovery of 1-[2-(2,4-dimethylphenylsulfanyl)phenyl]piperazine (Lu AA21004): a novel multimodal compound for the treatment of major depressive disorder. J Med Chem. 2011; 54(9): 3206-3221.

3. Pehrson AL, Cremers T, Bétry C, et al. Lu AA21004, a novel multimodal antidepressant, produces regionally selective increases of multiple neurotransmitters-a rat microdialysis and electrophysiology study. Eur Neuropsychopharmacol. 2013; 23(2): 133-145.

4. Sanchez C, Asin KE, Artigas F. Vortioxetine, a novel antidepressant with multimodal activity: review of preclinical and clinical data. Pharmacol Ther. 2015; 145: 43-57.

5. Mørk A, Montezinho LP, Miller S, et al. Vortioxetine (Lu AA21004), a novel multimodal antidepressant, enhances memory in rats. Pharmacol Biochem Behav. 2013; 105: 41-50.

6. Stahl SM. Modes and nodes explain the mechanism of action of vortioxetine, a multimodal agent (MMA): enhancing serotonin release by combining serotonin (5HT)transporter inhibition with action at 5HT receptors (5HT1A, 5HT1B, 5HT1D, 5HT7 receptors). CNS Spectr. 2015; 20(2): 93-97.

7. Stahl SM. Modes and nodes explain the mechanism of action of vortioxetine, a multimodal agent (MMA): modifying serotonin's downstream effects on glutamate and GABA (gamma-aminobutyric acid) release by blocking $5 \mathrm{HT} 3$ and $5 \mathrm{HT} 7$ receptors. CNS Spectr. In press.

8. Stahl SM. Modes and nodes explain the mechanism of action of vortioxetine, a multimodal agent (MMA): enhancing downstream release of neurotransmitters by blocking $5 \mathrm{HT} 3$ receptors. CNS Spectr. In press.

9. Insel T, Cuthbert B, Garvey M, et al. Research domain criteria (RDoC): toward a new classification framework for research on mental disorders. Am J Psychiatry. 2010; 167(7): 748-751.

10. Stahl SM. The last diagnostic and statistical manual (DSM): replacing our symptom-based diagnoses with a brain circuit-based classification of mental illnesses. CNS Spectr. 2013; 18(2): 65-68.

11. Stahl SM. Stahl's Essential Psychopharmacology. 4th ed. Cambridge, UK: Cambridge University Press; 2013.

12. Fink KB, Gothert M. 5HT receptor regulation of neurotransmitter release. Pharmacol Rev. 2007; 59(4): 360-417.

13. Pehrson AL, Sanchez C. Serotonergic modulation of glutamate neurotransmission as a strategy for treating depression and cognitive dysfunction. CNS Spectr. 2014; 19(2): 121-133.

14. Assié MB, Lomentach $\mathrm{H}$, Ravailhe V, Faucillon V, NewmanTancredi A. Rapid desensitization of somatodendritic 5HT1A 


\section{BRAINSTORMS-Clinical Neuroscience Update}

receptors by chronic administration of the high-efficacy 5HT1A agonist, F13714: a microdialysis study in the rat. Br J Pharmacol. 2006; 149(2): 170-178.

15. Gardier AM, Malgié I, Trillat AC, Jacquot C, Artigas F. Role of 5HT1A autoreceptors in the mechanism of action of serotoninergic antidepressant drugs: recent findings from in vivo microdialysis studies. Fundam Clin Pharmacol. 1996; 10(1): 16-27.

16. Romero L, Ben N, Artigas F, De Montigny C, Blier P. Effect of pindolol on the function of pre- and postsynaptic 5 HT1A receptors: in vivo microdialysis and electrophysiological studies in the rat brain. Neuropsychopharmacology. 1996; 15(4): 349-360.

17. Sprouse JS, Aghajanian GK. Electrophysiological responses of serotoninergic dorsal raphe neurons to 5HT1A and 5HT1B agonists. Synapse. 1987; 1(1): 3-9.

18. Izumi J, Washizuka M, Miura N, Hiraga Y, Ikeda Y. Hippocampal serotonin 5HT1A receptor enhances acetylcholine release in conscious rats. J Neurochem. 1994; 62(5): 1804-1808.

19. Consolo S, Ramponi S, Ladinsky H, Baldi G. A critical role for D1 receptors in the 5HT1A-mediated facilitation of in vivo acetylcholine release in rat frontal cortex. Brain Res. 1996; 707(2): 320-323.

20. Suzuki M, Matsuda T, Asano S, Somboonthum P, Takuma K, Baba A. Increase of noradrenaline release in the hypothalamus of freely moving rat by postsynaptic 5-hydroxytryptamine $1 \mathrm{~A}$ receptor activation. Br J Pharmacol. 1995; 115(4): 703-711.

21. Suwabe A, Kubota M, Niwa M, Kobayashi K, Kanba S. Effect of a 5HT1A receptor agonist, flesinoxan, on the extracellular noradrenaline level in the hippocampus and on the locomotor activity of rats. Brain Res. 2000; 858(2): 393-401.

22. Díaz-Mataix L, Scorza MC, Bortolozzi A, Toth M, Celada P, Artigas F. Involvement of 5HT1A receptors in prefrontal cortex in modulation of dopaminergic activity: role in atypical antipsychotic action. J Neurosci. 2005; 25(47): 10831-10843.

23. Alex KD, Pehak EA. Pharmacologic mechanisms of serotonergic regulation of dopamine neurotransmission. Pharmacol Ther. 2007; 113(2): 296-320.

24. Maura G, Raiteri M. Cholinergic terminals in rat hippocampus possess 5 HT1B receptors mediating inhibition of acetylcholine release. Eur J Pharmacol. 1986; 129(3): 333-337.

25. Maura G, Fedele E, Raiteri M. Acetylcholine release from rat hippocampal slices is mediated by 5-hydroxytryptamine. Eur J Pharmacol. 1989; 165(2-3): 173-179.

26. Johnson DE, Drummond E, Grimwood S, et al. The 5-hydroxytryptamine 4 receptor agonists prucalopride and PRX-03140 increase acetylcholine and histamine levels in the rat prefrontal cortex and the power of stimulated hippocampal theta oscillations. J Pharmacol Exp Ther. 2012; 341(3): 681-691.

27. Katona C, Hansen T, Olsen CK. A randomized, double blind, placebo controlled, duloxetine referenced, fixed dose study comparing the efficacy and safety of Lu AA21004 in elderly patients with major depressive disorder. Int Clin Psychopharmacol. 2012; 27(4): 215-223.

28. McIntyre RS, Lophaven S, Olsen C. A randomized, double-blind, placebo controlled study of vortioxetine on cognitive function in depressed adults. Int J Neuropsychopharmacol. 2014; 17(10): 1557-1567.

29. Mahableshwarkar AR, Zajecka J, Jacobson W, Chen Y, Keefe RSE. A randomized, placebo-controlled, active-reference, double blind, flexible dose study of the efficacy of vortioxetine on cognitive function in major depressive disorder. Neuropsychopharmacology. In press. DOI: $10.1038 /$ npp.2015.52. 